\title{
Simulation of Magnetohydrodynamic and Thermal Coupling in the Linear Induction MHD Pump
}

\author{
F.Z. Kadid ${ }^{1}$, S. Drid ${ }^{2}$ and R. Abdessemed ${ }^{1}$ \\ ${ }^{I}$ LEB Research Laboratory, Electrical Engineering Department, University of Batna, Rue \\ Chahid Med El Hadi Boukhlouf, 05000 Batna, Algeria \\ ${ }^{2}$ LSP-IE Research Laboratory, Electrical Engineering Department, University of Batna, Rue Chahid Med El Hadi \\ Boukhlouf, 05000 Batna, Algeria
}

Email: fzohra_kadid@hotmail.com,s_drid@yahoo.fr

(Received February 4, 2009; accepted September 21, 2009)

\begin{abstract}
This article is concerned with the study of a coupling between the stationary Maxwell equations, the transient state Navier Stokes and thermal equations. The model developed computes the magnetic field using the finite element method and calculates the velocity and the temperature using the finite volume method. The paper focuses on the analysis of the flux density, the electromagnetic thrust, the electric power density, the velocity, the pressure and the temperature in the channel of the MHD pump. Effect of the frequency is also presented.
\end{abstract}

Keywords: Magnetohydrodynamics (MHD), finite element method (FEM), finite volume method (FVM), streamvorticity formulation, temperature, linear induction MHD pump.

\section{NOMENCLATURE}

$\begin{array}{ll}\text { A } & \text { magnetic vector potential } \\ \mathbf{B} & \text { magnetic induction } \\ \mathbf{F} & \text { electromagnetic thrust } \\ C_{p} & \text { specific heat } \\ \mathrm{f} & \text { frequency } \\ \mathbf{J}_{\mathbf{e x}} & \text { excitation current density } \\ \mathbf{J}_{\mathbf{i}} & \text { induced eddy currents } \\ j & \text { imaginary } \\ \mathrm{K} & \text { thermal conductivity } \\ \mathrm{p} & \text { pressure of the fluid } \\ p_{s} & \text { thermal source (electric power density) } \\ \mathrm{T} & \text { temperature }\end{array}$

\section{INTRODUCTION}

Magnetohydrodynamics (MHD) is the study of the motion of electrically conducting fluids in the presence of magnetic fields. Effects from such interactions can be observed in liquids and gases. A number of researches have investigated the flow of an electrically conducting fluid through channels because of its important applications in MHD generators, pumps, accelerators, flowmeters and blood flow measurements (Berton 1991 and Vinsard et al. 1998). The pumping of

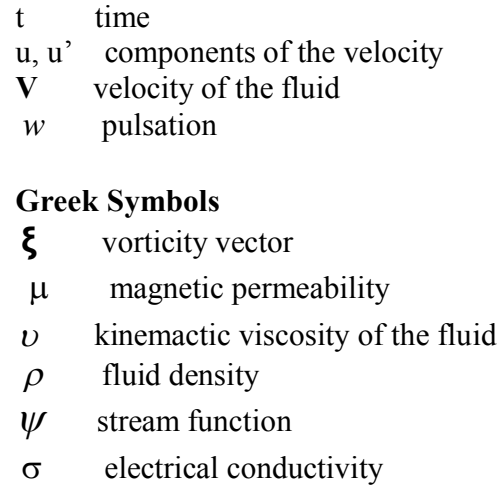

liquid metal may use an electromagnetic device, which induces eddy currents in the metal. These induced currents and their associated magnetic field generate the Lorentz force whose effect can be actually the pumping of the liquid metal. The advantage of these pumps which ensure the energy transformation is the absence of moving parts. Linear induction MHD pumps are electromagnetic devices using the principle of induction motors to move liquid metal by the action of a sliding field Takorabet et al. (2006). 
The magnetohydrodynamic problem is studied using the finite element method for the electromagnetic problem and the finite volume method for the hydrodynamic and thermal problems.

The difficulty in the electromagnetic problem is the presence of the convective term $\mathbf{V} \wedge \mathbf{B}$ due to the movement of the fluid, where $\mathbf{B}$ is the magnetic induction and $\mathbf{V}$ is the velocity (Kadid et al. 2003, $2004 a$, b). However, in the hydrodynamic study, the difficulty is that the incompressibility constraint given by $\operatorname{div} \mathbf{V}=0$ which couples the velocity $\mathbf{V}$ and the pressure $\mathrm{p}$ and also the choice of the numerical method.

Many formulations related to solve the incompressibility constraint in the Navier Stokes equations are given in the literature. These formulations are often referred as the pseudo-compressibility method Guemond et al. (2006) and the stream-vorticity formulation (Anderson 1984 and Krzeminski 2000). After simplifications of the Navier-Stokes equations, it is possible to obtain the velocity and the pressure, for that several methods are widely used in computational fluid dynamics such as finite difference method, the finite element method Nesliturk, Tezer-Sezgin (2006), the boundary element method Tezer-Sezgin, Han Aydin (2006) and the finite volume method Patankar (1980).

So, in this work we choose the stream-vorticity formulation which is the most used in hydrodynamics problems in the transient case and the finite volume method is employed to enforce such a local conservation property and it is easier comparing to the finite element method. The resolution of coupled equations is obtained by introducing the magnetic vector potential $\mathbf{A}$, the vorticity $\boldsymbol{\xi}$, the stream function $\psi$ and the temperature T. For that, it is initially necessary to determine the flux density then the induced currents, the electromagnetic thrust and the thermal source which allow calculating the velocity, the pressure and the temperature at any point of the channel. Finally, the analysis of the influence of the frequency on the fluid flow and on the temperature is presented.

\section{Electromagnetic Model}

\section{2 .1 Mathematical Formulation}

The cross section of the MHD pump in the (x-y) plan is shown in Fig. 1. It has two inductors which contain the coils, the air gap and the external area while the channel contains the liquid metal. The conducting fluid is assumed to be viscous and incompressible.

The Maxwell's equations applied to the MHD pump will give rise to the following equation:

$\operatorname{rot}\left(\frac{1}{\mu} \operatorname{rot} \mathbf{A}\right)+\sigma\left(\frac{\partial \mathbf{A}}{\partial t}-\mathbf{V} \times \operatorname{rot} \mathbf{A}\right)=\mathbf{J}_{\mathbf{e x}}$

where $\mathbf{A}$ is the magnetic vector potential, $\sigma$ is the electrical conductivity, $\mu$ the magnetic permeability, $\mathbf{V}$ is the velocity of the fluid and $\mathbf{J}_{\mathbf{e x}}$ the excitation current density.

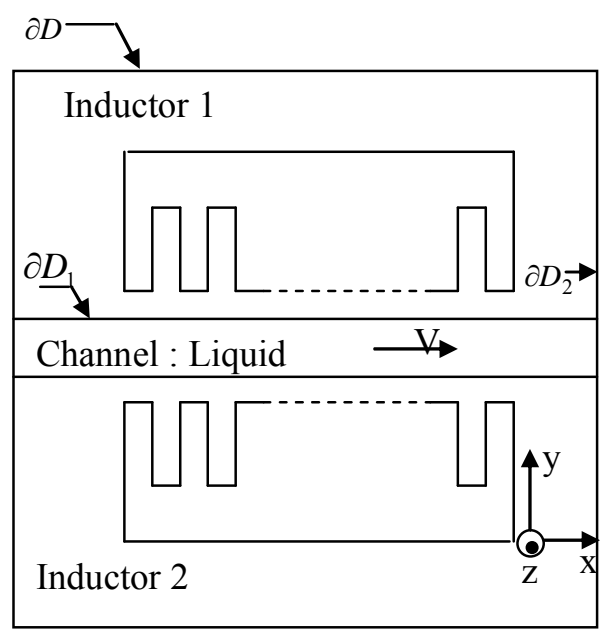

Fig. 1. Schematic view of the MHD pump

For the calculation reported in the following, mercury is considered as the fluid.

After developing the above equations in Cartesian coordinates and in sinusoidal mode, the final integrodifferential system with the Dirichlet boundary condition is:

$$
\begin{aligned}
& -\frac{1}{\mu}\left(\frac{\partial^{2} A}{\partial x^{2}}+\frac{\partial^{2} A}{\partial y^{2}}\right)+ \\
& +\sigma\left(j w A+V \frac{\partial A}{\partial x}\right)=J_{e x}
\end{aligned}
$$$$
A=0 \text { on } \partial D
$$

The currents of the windings generate the traveling magnetic field which produces a current in the liquid metal. As a consequence a Lorentz force acting on the fluid is obtained.

The eddy currents inside the channel are computed by:

$\mathbf{J}_{\mathbf{i}}=-\sigma\left(\frac{\partial \mathbf{A}}{\partial \mathrm{t}}-\mathbf{V} \times \operatorname{rot} \mathbf{A}\right)$

The thrusts are given by:

$\mathbf{F}=\mathbf{J}_{\mathbf{i}} \times \mathbf{r o t} \mathbf{A}$

\section{2 .2 Finite Element Method}

The electromagnetic Eq. (2) is solved by using the finite element method. We apply the weak Galerkine method to this equation which consists to seek $\mathrm{n}$ projection functions $\varphi_{1}, \varphi_{2}, \ldots, \varphi_{\mathrm{n}}$ such as:

$$
\begin{aligned}
\int_{\Omega} \varphi_{i} & {\left[\frac{1}{\mu}\left(\frac{\partial^{2} A}{\partial x^{2}}+\frac{\partial^{2} A}{\partial y^{2}}\right)\right] d \Omega+} \\
& +\int_{\Omega} \varphi_{i}\left(-\sigma j w A+J_{e x}-\sigma V \frac{\partial A}{\partial x}\right) d \Omega=0
\end{aligned}
$$


After evaluating the resulting integrals by parts over the whole problem domain $(\Omega)$ and then substituting the appropriate boundary conditions, we obtain a set of simultaneous partial differential equations of the form (Sadiku, 1992 and Jianming, 1993):

$$
\left[j \omega[C]+\left[M_{1}\right]+\left[M_{2}\right]\right][A]=[F]
$$

The matrices $[\mathrm{C}],\left[\mathrm{M}_{1}\right]$ and $\left[M_{2}\right]$ are calculated considering the element matrices, appropriate shape functions (Kadid et al. 2003, 2004a, b and Jianming 1993). The vector $[F]$ accounts for the current $J_{\text {ex }}$. The resulting equations are solved using the iterative method until convergence is reached. Once A is obtained, we can compute the magnetic induction field by using $\vec{B}=\operatorname{rot} \vec{A}$.

\section{THE HYDRODYNAMIC AND THERMAL MODELS}

\subsection{Navier Stokes Equations}

The evolution of the velocity in the transient state condition in the channel of the MHD pump is governed by the Navier Stokes equations such as Anderson (1984):

$$
\begin{aligned}
& \frac{\partial \mathbf{V}}{\partial t}+(\nabla . \mathbf{V}) \mathbf{V}=-\frac{1}{\rho} \nabla p+v \mathbf{\Delta} \mathbf{V}+\frac{\mathbf{F}}{\rho} \\
& \operatorname{div} \mathbf{V}=0
\end{aligned}
$$

where $\mathrm{p}$ the pressure of the fluid, $v$ the kinematic viscosity of the fluid, $\mathbf{F}$ the electromagnetic thrust and $\rho$ the fluid density.

The development of the equation of the flow in Cartesian coordinates gives:

$$
\left\{\begin{array}{l}
\frac{\partial u}{\partial t}+u \frac{\partial u}{\partial x}+u^{\prime} \frac{\partial u}{\partial y}=-\frac{1}{\rho} \frac{\partial p}{\partial x}+v\left(\frac{\partial^{2} u}{\partial x^{2}}+\frac{\partial^{2} u}{\partial y^{2}}\right)+\frac{F_{x}}{\rho} \\
\frac{\partial u^{\prime}}{\partial t}+u \frac{\partial u^{\prime}}{\partial x}+u^{\prime} \frac{\partial u^{\prime}}{\partial y}=-\frac{1}{\rho} \frac{\partial p}{\partial y}+v\left(\frac{\partial^{2} u^{\prime}}{\partial x^{2}}+\frac{\partial^{2} u^{\prime}}{\partial y^{2}}\right)+\frac{F_{y}}{\rho} \\
\frac{\partial u}{\partial x}+\frac{\partial u^{\prime}}{\partial y}=0
\end{array}\right.
$$

The boundary conditions are such as:

$$
\begin{cases}\mathrm{u}=\mathrm{u}^{\prime}=0 & \text { on } \partial \mathrm{D}_{1} \\ \frac{\partial \mathrm{u}}{\partial \mathrm{n}}=0, \frac{\partial \mathrm{u}^{\prime}}{\partial \mathrm{n}}=0 & \text { on } \partial \mathrm{D}_{2}\end{cases}
$$

The real difficulty is the calculation of the velocity lies in the unknown pressure. To overcome this difficulty is to relax the incompressibility constraint in an appropriate way. . So, the elimination of pressure from the equations leads to a vorticity-stream function
(Anderson, 1984 and Krzeminski, 2000). The vorticity vector is defined by:

$$
\xi=\operatorname{rot} V
$$

The stream function is given in 2D Cartesian coordinates as:

$\frac{\partial \psi}{\partial y}=u$

$\frac{\partial \psi}{\partial x}=-u^{\prime}$

where $\mathrm{u}$ and $\mathrm{u}$ ' the components of the velocity $\mathrm{V}$.

We eliminate the pressure from the Eq. (10) and we use the two new dependent variables $\xi$ and $\Psi$ to obtain the following equation:

$$
\begin{aligned}
\frac{\partial \xi}{\partial t}+ & u \frac{\partial \xi}{\partial x}+u^{\prime} \frac{\partial \xi}{\partial y}= \\
& =v\left(\frac{\partial^{2} \xi}{\partial x^{2}}+\frac{\partial^{2} \xi}{\partial y^{2}}\right)+\frac{1}{\rho}\left(\frac{\partial F_{y}}{\partial x}-\frac{\partial F_{x}}{\partial y}\right)
\end{aligned}
$$

After substituting Eq. (13) into Eq. (12) we obtain an equation involving the new dependant variables $\xi$ and $\psi$ such as:

$\frac{\partial^{2} \psi}{\partial x^{2}}+\frac{\partial^{2} \psi}{\partial y^{2}}=-\xi$

To determine the pressure, the resolution of an additional equation is necessary; the latter is obtained by differentiating Eq. (8) and using the continuity Eq. (9). This equation is referred to as the Poisson equation for pressure:

$$
\Delta p=2 \rho\left(\frac{\partial^{2} \psi}{\partial x^{2}} \frac{\partial^{2} \psi}{\partial y^{2}}\right)
$$

\subsection{Thermal problem}

The thermal phenomena are studied only in the channel of the MHD pump. So, the governing thermal equation is given by:

$$
\begin{aligned}
\rho C_{p} \frac{\partial T}{\partial t} & +\frac{T}{\rho}\left(\frac{\partial \rho}{\partial T}\right) p \frac{D p}{D t}= \\
& =\operatorname{div}(K \overrightarrow{\operatorname{grad} T})+p_{s}
\end{aligned}
$$

where $\rho$ is the density of the fluid, $C_{p}$ the specific heat, $\mathrm{K}$ the thermal conductivity, $\mathrm{T}$ the temperature and $p_{s}$ the thermal source (electric power density) induced by eddy current such as:

$p_{s}=\frac{J_{i}^{2}}{2 \sigma}$

After developments in Cartesian coordinates, replacing the source term $p_{s}$ and by neglecting the term of 
pressure; this is due to the low velocities obtained in the hydrodynamic study, we obtain:

$\rho C_{p} \frac{\partial T}{\partial t}=K\left[\frac{\partial^{2} T}{\partial x^{2}}+\frac{\partial^{2} T}{\partial y^{2}}\right]+\frac{J_{i} J_{i}^{*}}{2 \sigma}$

$J_{i}^{*}$ is the conjugate of $J_{i}$

The boundary conditions (Dirichlet and Neumann) applied to the channel (Fig. 1) are given by:

$\mathrm{T}=298^{\circ} \mathrm{K}$ on $\partial \mathrm{D}_{1}$.

And $\frac{\partial T}{\partial n}=0$ on $\partial \mathrm{D}_{2}$.

\subsection{Numerical Procedure}

For the model of the fluid flow, there is one control volume surrounding each node (Fig. 2) and the differential Eq. (14) is integrated over each control volume using the finite volume approach Patankar (1980):

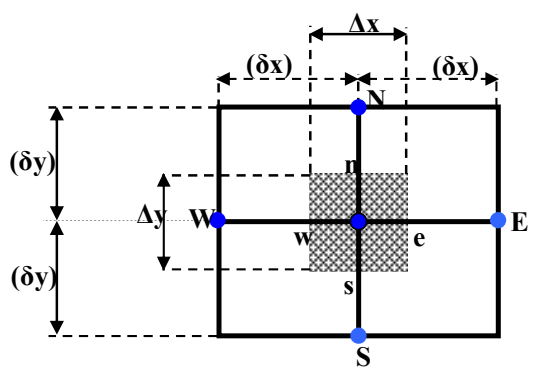

Fig. 2. Discretisation in finite volume method

$$
\int_{\Omega}\left(\begin{array}{l}
\frac{\partial \xi}{\partial t}+u \frac{\partial \xi}{\partial x}+u^{\prime} \frac{\partial \xi}{\partial y}-v\left(\frac{\partial^{2} \xi}{\partial x^{2}}+\frac{\partial^{2} \xi}{\partial y^{2}}\right) \\
-\frac{1}{\rho}\left(\frac{\partial F_{y}}{\partial x}-\frac{\partial F_{x}}{\partial y}\right)
\end{array}\right) d \Omega=0
$$

The discretisation equation results in a series of discrete algebraic equations that take the form:

$$
a_{P} \xi_{P}=\sum a_{n b} \xi_{n b}+b
$$

where $a_{p}$ terms are the active coefficients on $\xi$, and "nb" implies summation over the neighboring nodes (those to the West, W; East, E; South, S; and North, N; of $\mathrm{P}$ for two-dimensional computations and $\mathrm{b}$ the source terms.

The code generated is based on an unstructured meshgeneration. The nodes of the mesh for the coupling model electromagnetic-hydrodynamic are the same in the channel.

At each time step electromagnetic and hydrodynamic problem can be solved alternatively and iteratively until convergence is reached.
We use the same steps for the thermal Eq. (19) as for the hydrodynamic Eq. (14):

$$
\begin{aligned}
& \int_{t}^{t+\Delta t} \int_{s}^{n} \int_{w}^{e} \rho C_{p} \frac{\partial T}{\partial t} d x d y d t \\
& =\int_{t}^{t+\Delta t} \int_{s}^{n} \int_{w}^{e}\left[K\left[\frac{\partial^{2} T}{\partial x^{2}}+\frac{\partial^{2} T}{\partial y^{2}}\right]+\frac{J_{i} J_{i}^{*}}{2 \sigma}\right] d x d y d t
\end{aligned}
$$

with the source expression, the discretisation equation would still look like Eq. (21), but the coefficients would change. The new set is:

$a_{p}^{\prime} T_{P}=\sum a_{n b}^{\prime} T_{n b}+b^{\prime}$

where the subscript "nb" denotes a neighbor, and the summation is to be taken over all the neighbors.

\section{Simulations AND RESUlts}

The potential vector $\vec{A}$ is calculated for each finite element node, by means of finite element method. Hydrodynamic and thermal calculations supply respectively the velocity, the pressure components and the temperature which must be known at each integration point of the finite volume method.

For the coupling of the two methods F.E.M - F.V.M, it is necessary to ensure an adaptation of the grid mesh, i.e. we must find the same nodes for the two different methods. The electromagnetic force density calculated by the finite element method is introduced in the hydrodynamic equations which are solved using the finite volume method to determine the velocity and the pressure of the fluid in the channel. Also, the thermal source is calculated for the thermal problem.

Figure 3 represents the coupling of the three problems such as: hydrodynamic-electromagnetic-thermal and it is divided into the following three parts.

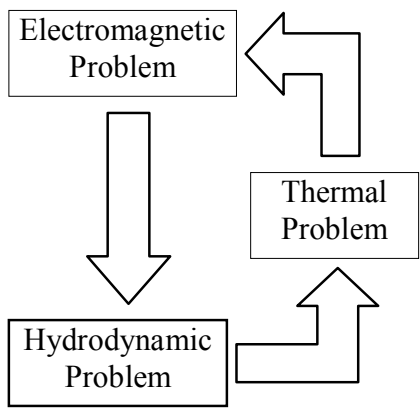

Fig. 3. The coupling scheme

- finite element method computation in the sinusoidal mode of the magnetic vector potential $\mathbf{A}$ by Eq. (2) and the computation of the force density by Eq. (5);

- finite volume method computation of the velocity in the transient state only in the channel of the MHD pump by using the vorticity vector-stream 
function Eq. (14). Once the stream function is determined, we can calculate the pressure at any point of the channel by the Eq. (16);

- finite volume method computation of the temperature in the transient state only in the channel of the MHD pump where the temperature is determined by the Eq. (19).

For the electromagnetic problem the whole MHD pump is meshed but in the hydrodynamic problem only the channel which contains the mercury liquid metal is meshed. The iterations are repeated until the error is low. As results Fig. 4 and Fig. 5 represent respectively the magnetic induction and the distribution of the electromagnetic thrust in the MHD pump, this force is the same as that obtained by Takorabet et al. (2006).

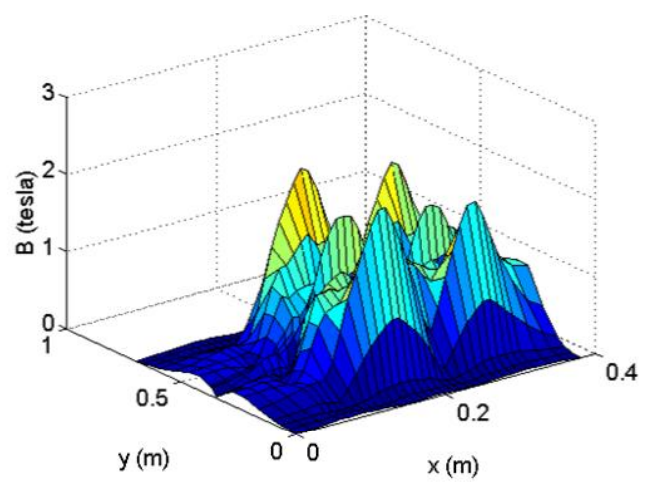

Fig . 4. The magnetic induction in the MHD pump

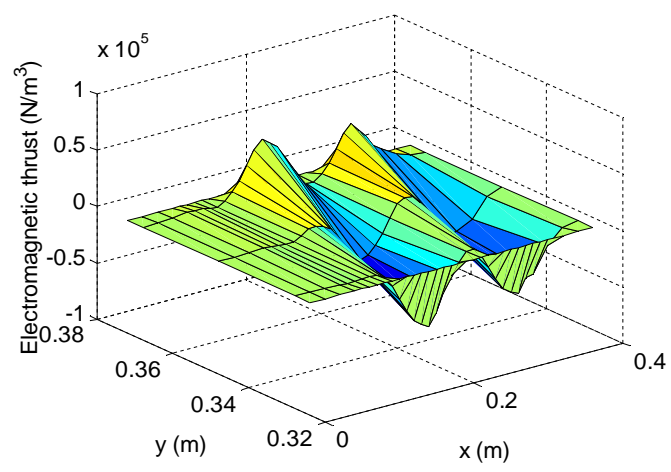

Fig. 5. The electromagnetic thrust

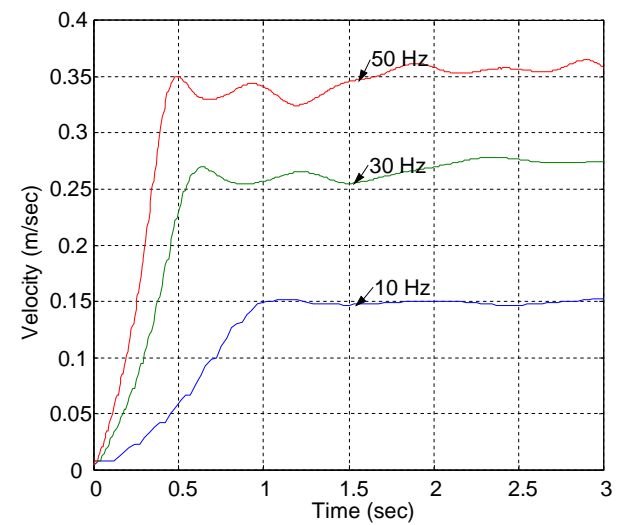

Fig. 6. Velocity in the middle of the channel for several frequencies

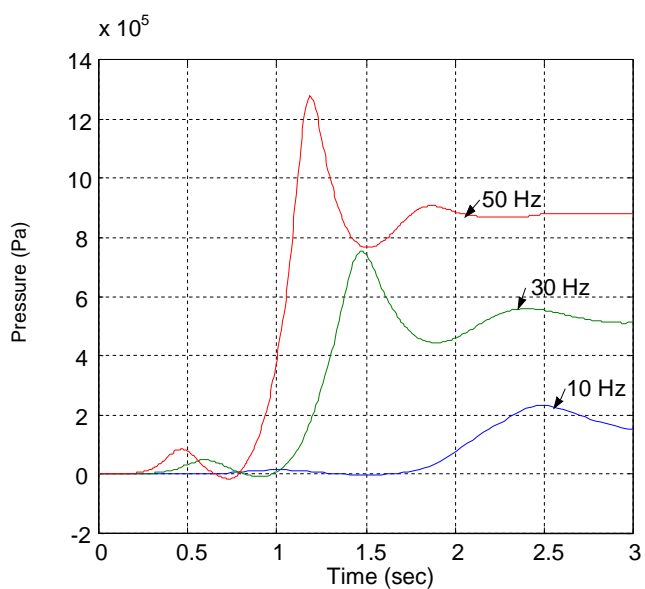

Fig. 7. Pressure in the middle of the channel for several frequencies

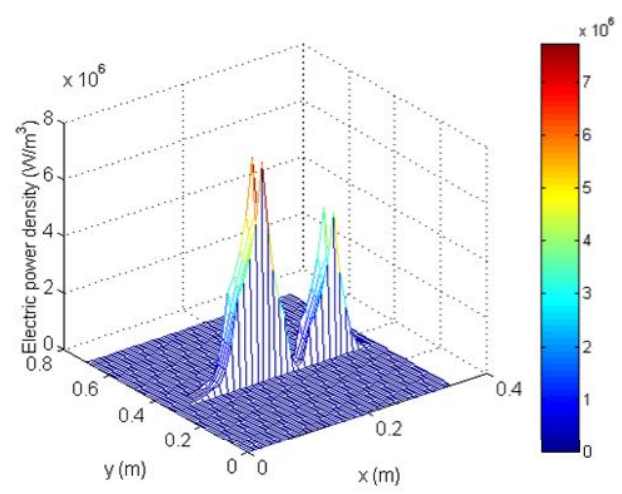

Fig. 8. The electric power density in the channel

Figure 6 represents the variation of the velocity of the pump for several frequencies. It is shown that the velocity increases as the frequency increases and the steady state is obtained approximately after two seconds.

Figure 7 shows the pressure variations for several frequencies. It is found that the pressure increases as the frequency increases. It is important to notice that the amplitudes of the pressure oscillations increase with the increasing of the frequency. Moreover, the "shock" values become more significant with a shorter transient state.

Figure 8 shows the electric power density in the channel. The maximum induced power reaches $610^{6} \mathrm{~W} / \mathrm{m}^{3}$. The pace obtained is directly related to that of the eddy current density. This characteristic of the heat source is used in the numerical calculation of the temperature.

Figure 9 shows the distribution of the temperature for different frequencies. We note that the temperature increase with the frequency. The maximum temperature for $\mathrm{f}=50 \mathrm{~Hz}$ reaches $372^{\circ} \mathrm{K}$. 


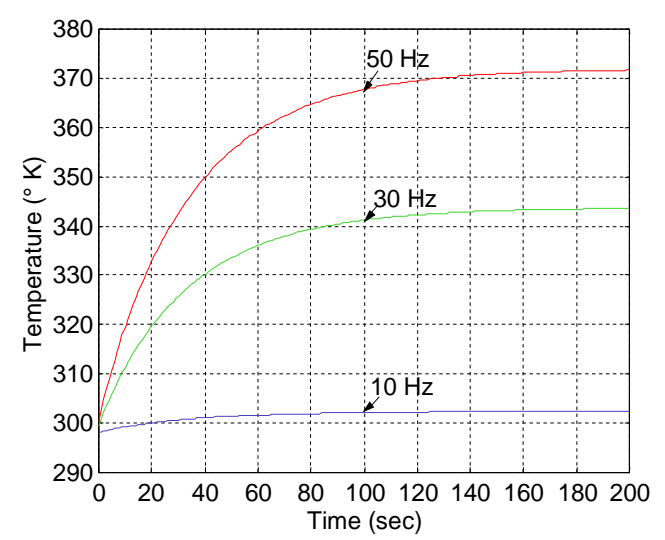

Fig. 9. Temperature in the channel for different frequencies

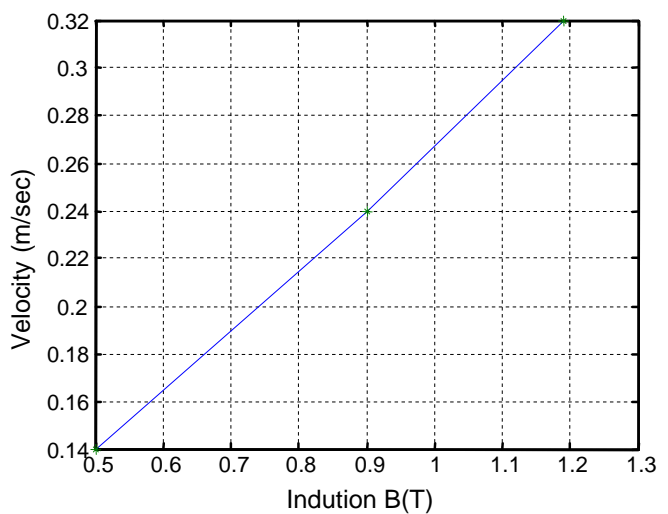

Fig. 10. Velocity of the fluid various inductions

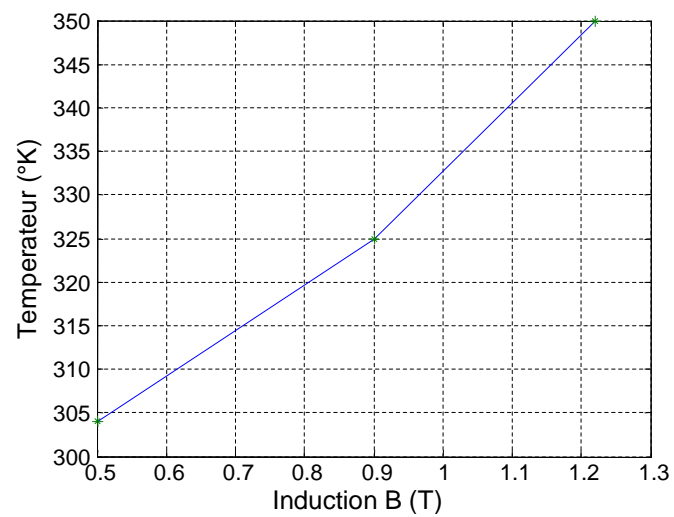

Fig. 11. Temperature of the fluid various inductions

The effect of the magnetic field on velocity and temperature are represented in Figs. 10 and 11. It is shown that the velocity and the temperature increase linearly with the magnetic field.

\section{Conclusion}

In this paper we have studied the coupled magnetohydrodynamic and thermal problems using $2 \mathrm{D}$ finite element-finite volume method taking into account the movement of the fluid. The results show the presence of fast transients and the oscillatory behavior in both the velocity and the pressure.
The obtained results confirm an influence of the frequency on the velocity and pressure distribution in the investigated flow (Yamagushi 2001; Affanni and Chiorboli 2006).

A visualization of the temperature curve leads to appreciate the effect of the frequency on the temperature in the channel and the obtained results of the temperature are identical to the results of Ghassemi and Passandeh (2003).

\section{REFERENCES}

Affanni, A. and G. Chiorboli (2006, April). Numerical modeling and experimental study of an $\mathrm{AC}$ magnetohydrodynamic (MHD) pump. IMTCInstrumentation and Measurement Technologie, Conference Sorento, Italy, IEEE, 2249-2253.

Anderson, D.A., J.C. Tannehill and R.H. Pletcher (1984). Computational Fluid Mechanics and Heat Transfer. Hemisphere Publishing Corporation.

Berton, R. (1991). Magnetohydrodynamique. Editions Masson.

Guermond, J.L., P. Minev and Jie Shen (2006). An overview of projection methods for incompressible flows. Computer methods in applied mechanics and engineering 195, ELSEVIER, 6011-6045.

Ghassemi, M. and R. Pasandeh (2003, May). Thermal and Electromagnetic analysis of an Electromagnetic launcher. IEEE Transactions On Magnetics 39(3), 1819-1822.

Jianming, J. (1993). The Finite Element Method in Electromagnetics. John Wiley and Sons.

Krzeminski, S.K., M. Smialek and M. Wlodarczyk (2000, July). Numerical Analysis of Peristaltic MHD flows. IEEE Transactions on Magnetics 36(4), 1319-1324.

Kadid, F.Z., R. Abdessemed and S. Drid (2003, December). Characterisation of 2D Eddy Currents in the Channel of Linear Induction MHD Pump. Journal of Electrical Engineering 3(2), 28-33, Romania.

Kadid, F.Z., R. Abdessemed and S. Drid (2004). 2D FEM modeling of the linear induction MHD Pump taking into account the movement of the fluid. AMSE Journals 73(4), 61-68.

Nesliturk, A.I. and M. Tezer-Sezgin M (2006). Finite element method of electrically driven magnetohydrodynamic flow. Journal of Computational and Applied Mathematics 192, ELSEVIER, 339-352.

Patankar, S.V (1980). Numerical Heat Transfer Fluid Flow. Hemisphere Publishing Corporation. 
F.Z. Kadid et al. / JAFM, Vol. 4, No. 1, 51-57, 2011.

Sadiku, M.N.O. (1992). Numerical Techniques in Electromagnetics. CRC Press.

Tezer-Sezgin, M. and S. Han Aydin (2006). Solution of magnetohydrodynamic flow problems using the boundary element method. Engineering Analysis with Boundary Elements 30, ELSEVIER, 411-418.

Takorabet, N. (2006, March). Computation of force density inside the channel of an electromagnetic pump by hermite projection. IEEE Transactions on Magnetics 42(3), 430-433.

Verardi, S.L.L., J.R. Cardoso and M.C. Costa (2001, September). Three dimensional finite element analysis of MHD duct flow by the penalty function formulation. IEEE Transactions on Magnetics 37(5), 3384-3387.

Vinsard, G., B. Laporte and N. Takorabet (1998, September). An analysis of the rotational forces in the secondary of an electromagnetic pump. IEEE Transactions on Magnetics 34(5), 3552-3555.

Yamagushi, T., Y. Kawase, M. Yoshida, Y. Saito and Y. Ohdachi (2001, September). 3-D finite element analysis of a linear induction motor. IEEE Transactions on Magnetics 37(5), 3668-3671. 